\title{
Boundedness of fractional integrals on weighted Herz spaces with variable exponent
}

\author{
Mitsuo Izuki ${ }^{*}$ and Takahiro Noi ${ }^{2}$
}

${ }^{\text {*Correspondence: }}$

izuki@okayama-u.ac.jp

${ }^{1}$ Faculty of Education, Okayama University, 3-1-1 Tsushima-naka,

Okayama, 700-8530, Japan

Full list of author information is

available at the end of the article

\section{Springer}

\begin{abstract}
Our aim is to prove the boundedness of fractional integral operators on weighted Herz spaces with variable exponent. Our method is based on the theory on Banach function spaces and the Muckenhoupt theory with variable exponent.
\end{abstract}

MSC: $42 B 35$

Keywords: Herz spaces; Muckenhoupt weight; variable exponent

\section{Introduction}

The boundedness of fractional integrals on function spaces is one of the important problems not in harmonic analysis but also in potential theory and in partial differential equations. Among the development of variable exponent analysis the we can list up boundedness of fractional integrals on function spaces with variable exponent. Capone, CruzUribe and Fiorenza [1] have proved the boundedness on Lebesgue spaces with variable exponent, provided that the exponents satisfy the log-Hölder continuous conditions. The conditions on variable exponents have been established by the study of the boundedness of the Hardy-Littlewood maximal operator on spaces with variable exponent [2-8]. CruzUribe, Fiorenza, Martell and Pérez [9] have also proved the boundedness of fractional integrals by virtue of the extrapolation method.

Every Herz space has an interesting norm involving both local and global information and has been studied in harmonic analysis. Lu and Yang [10] have proved the boundedness of fractional integrals on Herz spaces. Inspired by the study of variable exponent analysis and on Herz spaces the first author has defined Herz spaces with variable exponent $[11,12]$. Later he has proved the boundedness of fractional integrals on Herz spaces with variable exponent [13]. Almeida and Drihem [14] have also independently proved the boundedness.

Based on the Muckenhoupt theory [15] the modern harmonic analysis has been greatly developed. Recently the generalized Muckenhoupt weights with variable exponent have been considered [16-20]. In particular, Cruz-Uribe, Fiorenza and Neugebauer [17] and Diening and Hästö [18] have independently proved the equivalence between the Muckenhoupt condition and the boundedness of the Hardy-Littlewood maximal operator on weighted Lebesgue spaces in the variable exponent setting. We also note that CruzUribe and Wang [21] have obtained the boundedness of fractional integrals on weighted Lebesgue spaces with variable exponent applying the extrapolation theorem.

(c) 2016 Izuki and Noi. This article is distributed under the terms of the Creative Commons Attribution 4.0 International License (http://creativecommons.org/licenses/by/4.0/), which permits unrestricted use, distribution, and reproduction in any medium, provided you give appropriate credit to the original author(s) and the source, provide a link to the Creative Commons license, and indicate if changes were made. 
In this paper we define weighted Herz spaces with variable exponent and prove the boundedness of fractional integrals on those spaces under proper assumptions on weights and exponents. Our argument is based on the theory on Banach function spaces and on the Muckenhoupt theory with variable exponent. The authors have also considered other problems on boundedness of some operators on weighted Herz spaces with variable exponent in the recent preprints [22, 23].

In this paper we use the following symbols and notation:

1. For any measurable set $E,|E|$ denotes the Lebesgue measure and $\chi_{E}$ means the characteristic function.

2. A locally integrable and positive function defined on $\mathbb{R}^{n}$ is said to be a weight. We write $w(E):=\int_{E} w(x) \mathrm{d} x$ for a weight $w$ and a measurable set $E$.

3. If there exists a positive constant $C$ independent of the main parameters such that $A \leq C B$, then we write $A \lesssim B$.

\section{Preliminaries}

\subsection{Variable Lebesgue spaces}

Based on the fundamental papers and books $[2,7,8,24]$ we introduce Lebesgue spaces with variable exponent. Let $p(\cdot): \mathbb{R}^{n} \rightarrow[1, \infty)$ be a measurable function. The variable exponent Lebesgue space $L^{p(\cdot)}\left(\mathbb{R}^{n}\right)$ is the set of all complex-valued measurable functions $f$ defined on $\mathbb{R}^{n}$ satisfying

$$
\rho_{p}(f):=\int_{\mathbb{R}^{n}}|f(x)|^{p(x)} \mathrm{d} x<\infty .
$$

It is well known that the variable exponent Lebesgue space $L^{p(\cdot)}\left(\mathbb{R}^{n}\right)$ becomes a Banach space equipped with a norm given by

$$
\|f\|_{L^{p(\cdot)}}:=\inf \left\{\lambda>0: \rho_{p}\left(\frac{f}{\lambda}\right) \leq 1\right\} .
$$

Denote by $\mathcal{P}\left(\mathbb{R}^{n}\right)$ the set of all measurable functions $p(\cdot): \mathbb{R}^{n} \rightarrow(1, \infty)$ such that

$$
1<p^{-}:=\underset{x \in \mathbb{R}^{n}}{\operatorname{essin}} p(x), \quad p^{+}:=\underset{x \in \mathbb{R}^{n}}{\operatorname{ess} \sup } p(x)<\infty .
$$

A measurable function $p(\cdot)$ defined on $\mathbb{R}^{n}$ is said to be globally log-Hölder continuous if it satisfies

$$
\begin{aligned}
& |p(x)-p(y)| \lesssim \frac{1}{-\log (|x-y|)} \quad\left(x, y \in \mathbb{R}^{n},|x-y| \leq 1 / 2\right), \\
& \left|p(x)-p_{\infty}\right| \lesssim \frac{1}{\log (e+|x|)} \quad\left(x \in \mathbb{R}^{n}\right)
\end{aligned}
$$

for some real number $p_{\infty}$. The set of $p(\cdot)$ satisfying (2) and (3) is denoted by $L H\left(\mathbb{R}^{n}\right)$. It is also well known that the Hardy-Littlewood maximal operator $M$, defined by

$$
M f(x):=\sup _{B: \text { ball, }, x \in B} \frac{1}{|B|} \int_{B}|f(y)| \mathrm{d} y
$$

is bounded on $L^{p(\cdot)}\left(\mathbb{R}^{n}\right)$ whenever $p(\cdot) \in \mathcal{P}\left(\mathbb{R}^{n}\right) \cap L H\left(\mathbb{R}^{n}\right)[3-6]$. 


\subsection{The Muckenhoupt weights with variable exponent}

Let $p(\cdot) \in \mathcal{P}\left(\mathbb{R}^{n}\right)$ and $w$ be a weight. The weighted variable exponent Lebesgue space $L^{p(\cdot)}(w)$ is the set of all complex-valued measurable functions $f$ such that $f w^{1 / p(\cdot)} \in L^{p(\cdot)}\left(\mathbb{R}^{n}\right)$. The space $L^{p(\cdot)}(w)$ is a Banach space equipped with the norm

$$
\|f\|_{L^{p(\cdot)}(w)}:=\left\|f w^{1 / p(\cdot)}\right\|_{L^{p(\cdot)}} .
$$

Below $p^{\prime}(\cdot)$ is the conjugate exponent of $p(\cdot)$ given by $1 / p(\cdot)+1 / p^{\prime}(\cdot)=1$. Now we define the Muckenhoupt classes. We begin with the classical Muckenhoupt $A_{1}$ weight.

Definition 1 A weight is said to be a Muckenhoupt $A_{1}$ weight if $M w(x) \lesssim w(x)$ holds for almost every $x \in \mathbb{R}^{n}$. The set $A_{1}$ consists of all Muckenhoupt $A_{1}$ weights.

The original Muckenhoupt $A_{p}$ class with constant exponent $p \in(1, \infty)$ established by Muckenhoupt [15] can be generalized in terms of a variable exponent as follows.

Definition 2 Suppose $p(\cdot) \in \mathcal{P}\left(\mathbb{R}^{n}\right)$. A weight $w$ is said to be an $A_{p(\cdot)}$ weight if

$$
\sup _{B: \text { ball }} \frac{1}{|B|}\left\|w^{1 / p(\cdot)} \chi_{B}\right\|_{L^{p(\cdot)}}\left\|w^{-1 / p(\cdot)} \chi_{B}\right\|_{L^{p^{\prime}(\cdot)}}<\infty
$$

Our symbol $A_{p(\cdot)}$ slightly differs from that in $[17,18,21]$ where the space $L^{p(\cdot)}(w)$ is defined as the set consisting of all $f$ such that $f w \in L^{p(\cdot)}\left(\mathbb{R}^{n}\right)$. If $p(\cdot)$ equals a constant $p \in(1, \infty)$ in Definition 2, then we see immediately that the definition is equivalent to the classical Muckenhoupt class $A_{p}$ [15].

Diening and Hästö [18] have pointed out that Definition 2 does not directly imply the monotone property of the class $A_{p(\cdot)}$. In order to obtain the property they have generalized the Muckenhoupt class as follows.

Definition 3 Suppose $p(\cdot) \in \mathcal{P}\left(\mathbb{R}^{n}\right)$. A weight $w$ is said to be an $\tilde{A}_{p(\cdot)}$ weight if

$$
\sup _{B: \text { ball }}|B|^{-p_{B}}\left\|w \chi_{B}\right\|_{L^{1}}\left\|w^{-1} \chi_{B}\right\|_{L^{p^{\prime}(\cdot) / p(\cdot)}}<\infty
$$

where $p_{B}:=\left(\frac{1}{|B|} \int_{B} \frac{1}{p(x)} \mathrm{d} x\right)^{-1}$ is the harmonic average of $p(\cdot)$ over $B$. The set $\tilde{A}_{p(\cdot)}$ consists of all $\tilde{A}_{p(\cdot)}$ weights.

Based on the definition $\tilde{A}_{p(\cdot)}$ Diening and Hästö [18], Lemma 3.1, have proved the next monotone property.

Theorem 1 Suppose $p(\cdot), q(\cdot) \in \mathcal{P}\left(\mathbb{R}^{n}\right) \cap L H\left(\mathbb{R}^{n}\right)$ and $p(\cdot) \leq q(\cdot)$. Then we have $A_{1} \subset \tilde{A}_{p(\cdot)} \subset$ $\tilde{A}_{q(\cdot)}$.

We next state the relation between the generalized Muckenhoupt conditions and the boundedness of the Hardy-Littlewood maximal operator on weighted Lebesgue spaces in the variable exponent setting.

Theorem 2 Suppose $p(\cdot) \in \mathcal{P}\left(\mathbb{R}^{n}\right) \cap L H\left(\mathbb{R}^{n}\right)$. Then the following three conditions are equivalent: 
(A) $w \in A_{p(\cdot)}$.

(B) $w \in \tilde{A}_{p(\cdot) \text {. }}$

(C) The Hardy-Littlewood maximal operator is bounded on the weighted variable Lebesgue space $L^{p(\cdot)}(w)$.

Cruz-Uribe, Fiorenza and Neugebauer [17] have proved $(A) \Leftrightarrow(C)$. On the other hand, Diening and Hästö [18] have proved $(\mathrm{B}) \Leftrightarrow(\mathrm{C})$. By Theorem 2 we can identify $A_{p(\cdot)}$ and $\tilde{A}_{p(\cdot)}$, provided that $p(\cdot) \in \mathcal{P}\left(\mathbb{R}^{n}\right) \cap L H\left(\mathbb{R}^{n}\right)$. Combining Theorems 1 and 2 we get the monotone property for the class $A_{p(\cdot)}$, that is, the next corollary is true.

Corollary 1 If $p(\cdot), q(\cdot) \in \mathcal{P}\left(\mathbb{R}^{n}\right) \cap L H\left(\mathbb{R}^{n}\right)$ and $p(\cdot) \leq q(\cdot)$, then we have

$$
A_{1} \subset A_{p(\cdot)} \subset A_{q(\cdot)}
$$

In order to state the boundedness of fractional integrals on weighted function spaces we shall define the class $A\left(p_{1}(\cdot), p_{2}(\cdot)\right)$ as follows.

Definition 4 Let $0<\beta<n$ and $p_{1}(\cdot), p_{2}(\cdot) \in \mathcal{P}\left(\mathbb{R}^{n}\right)$ such that $1 / p_{2}(x) \equiv 1 / p_{1}(x)-\beta / n$. A weight $w$ is said to be an $A\left(p_{1}(\cdot), p_{2}(\cdot)\right)$ weight if

$$
\left\|w \chi_{B}\right\|_{L^{p_{2}(\cdot)}}\left\|w^{-1} \chi_{B}\right\|_{L^{p_{1}^{\prime}(\cdot)}} \lesssim|B|^{1-\frac{\beta}{n}}
$$

holds for all balls $B \subset \mathbb{R}^{n}$.

Lemma 1 Let $0<\beta<n$ and $p_{1}(\cdot), p_{2}(\cdot) \in \mathcal{P}\left(\mathbb{R}^{n}\right)$ such that $1 / p_{2}(x) \equiv 1 / p_{1}(x)-\beta / n$. Then $w \in A\left(p_{1}(\cdot), p_{2}(\cdot)\right)$ if and only if $w^{p_{2}(\cdot)} \in A_{1+p_{2}(\cdot) / p_{1}^{\prime}(\cdot)}$.

Proof Cruz-Uribe and Wang [21], Proposition 5.4, have proved a result similar to the lemma. Komori and Matsuoka [25] have also considered the case with constant exponent. We will prove the lemma by referring to [21, 25].

Note that

$$
\frac{p_{2}(\cdot)}{1+\frac{p_{2}(\cdot)}{p_{1}^{\prime}(\cdot)}}=\frac{1}{\frac{1}{p_{2}(\cdot)}+\frac{1}{p_{1}^{\prime}(\cdot)}}=\frac{1}{1-\frac{\beta}{n}}
$$

by the assumption on $p_{1}(\cdot)$ and $p_{2}(\cdot)$. Below we fix a ball $B \subset \mathbb{R}^{n}$ arbitrarily. It is easy to see that

$$
\begin{aligned}
& \frac{1}{|B|}\left\|\left(w^{p_{2}(\cdot)}\right)^{\left(1+\frac{p_{2}(\cdot)}{p_{1}^{\prime}(\cdot)}\right)^{-1}} \chi_{B}\right\|_{L}{ }_{L}+\frac{p_{2}(\cdot)}{p_{1}^{\prime}(\cdot)}\left\|\left(w^{p_{2}(\cdot)}\right)^{-\left(1+\frac{p_{2}(\cdot)}{p_{1}^{\prime}(\cdot)}\right)^{-1}} \chi_{B}\right\|_{L}\left(1+\frac{p_{2}(\cdot)}{p_{1}^{\prime}(\cdot)}\right)^{\prime} \\
& =\frac{1}{|B|}\left\|w^{p_{2}(\cdot)\left(1+\frac{p_{2}(\cdot)}{p_{1}^{\prime} \cdot(\cdot)}\right)^{-1}} \chi_{B}\right\|_{L}^{1+\frac{p_{2}(\cdot)}{p_{1} \cdot(\cdot)}}\left\|w^{-p_{2}(\cdot)\left(1+\frac{p_{2}(\cdot)}{p_{1}^{\prime} \cdot(\cdot)}\right)^{-1}} \chi_{B}\right\|_{L}{ }_{L}^{\left(1+\frac{p_{2}(\cdot)}{p_{1}^{\prime}(\cdot)}\right)^{\prime}} \\
& =\frac{1}{|B|}\left\|w \chi_{B}\right\|_{L^{p_{2}(\cdot)}}^{\frac{1}{1-\beta / n}}\left\|w^{-1} \chi_{B}\right\|_{L^{p_{1}^{\prime}(\cdot)}}^{\frac{1}{1-\beta / n}}
\end{aligned}
$$

holds. 
If $w \in A\left(p_{1}(\cdot), p_{2}(\cdot)\right)$, then we have

$$
\begin{aligned}
& \frac{1}{|B|}\left\|\left(w^{p_{2}(\cdot)}\right)^{\left(1+\frac{p_{2}(\cdot)}{p_{1}^{\prime}(\cdot)}\right)^{-1}} \chi_{B}\right\|_{L}{ }_{L}+\frac{p_{2}(\cdot)}{p_{1}^{\prime}(\cdot)}\left\|\left(w^{p_{2}(\cdot)}\right)^{-\left(1+\frac{p_{2}(\cdot)}{p_{1}^{\prime}(\cdot)}\right)^{-1}} \chi_{B}\right\|_{\left.L_{\left(1+\frac{p_{2}(\cdot)}{p_{1}^{\prime}(\cdot)}\right.}\right)^{\prime}} \\
& \quad=\frac{1}{|B|}\left\|w \chi_{B}\right\|_{L^{p_{2}(\cdot)}}^{\frac{1}{1-\beta / n}}\left\|w^{-1} \chi_{B}\right\|_{L^{p_{1}^{\prime}(\cdot)}}^{\frac{1}{1-\beta / n}} \lesssim 1 .
\end{aligned}
$$

This implies that $w^{p_{2}(\cdot)} \in A_{1+\frac{p_{2}(\cdot)}{p_{1}^{\prime}(\cdot)}}$.

$$
\begin{aligned}
& \text { If } w^{p_{2}(\cdot)} \in A_{1+\frac{p_{2}(\cdot)}{p_{1}^{\prime}(\cdot)}} \text {, then, by }(4) \text {, we see that } \\
& \qquad \begin{array}{l}
\left\|w \chi_{B}\right\|_{L^{p_{2}(\cdot)}}\left\|w^{-1} \chi_{B}\right\|_{L^{p_{1}^{\prime}(\cdot)}} \\
\quad=\|\left(w^{p_{2}(\cdot)}\right)^{\left(1+\frac{p_{2}(\cdot)}{p_{1}^{\prime}(\cdot)}\right)^{-1} \chi_{B}\left\|_{L}^{1-\beta / n}{ }_{L}^{1+\frac{p_{2}(\cdot)}{p_{1}^{\prime}(\cdot)}}\right\|\left(w^{p_{2}(\cdot)}\right)^{-\left(1+\frac{p_{2}(\cdot)}{p_{1}^{\prime}(\cdot)}\right)^{-1}} \chi_{B} \|_{L}^{1-\beta / n}{ }_{L}^{\left(1+\frac{p_{2}(\cdot)}{p_{1}^{\prime}(\cdot)}\right)^{\prime}}} \\
\lesssim|B|^{1-\beta / n}
\end{array}
\end{aligned}
$$

holds. Hence we have $w \in A\left(p_{1}(\cdot), p_{2}(\cdot)\right)$. This completes the proof.

\subsection{Herz spaces with variable exponent}

Let $\Omega \subset \mathbb{R}^{n}$ be a measurable set and $w$ a positive and locally integrable function on $\Omega$. The set $L_{\text {loc }}^{p(\cdot)}(\Omega, w)$ consists of all functions $f$ satisfying the following condition: for all compact sets $E \subset \Omega$ there exists a constant $\lambda>0$ such that

$$
\int_{E}\left|\frac{f(x)}{\lambda}\right|^{p(x)} w(x) \mathrm{d} x<\infty
$$

Let $l \in \mathbb{Z}$. We use the following notations in order to define Herz spaces:

$$
B_{l}:=\left\{x \in \mathbb{R}^{n}:|x| \leq 2^{l}\right\}, \quad R_{l}:=B_{l} \backslash B_{l-1}, \quad \chi_{l}:=\chi_{R_{l}} .
$$

Definition 5 Let $p(\cdot) \in \mathcal{P}\left(\mathbb{R}^{n}\right), 0<q<\infty$, and $\alpha \in \mathbb{R}$. The homogeneous weighted Herz space $\dot{K}_{p(\cdot)}^{\alpha, q}(w)$ is the collection of $f \in L_{\text {loc }}^{p(\cdot)}\left(\mathbb{R}^{n} \backslash\{0\}, w\right)$ such that

$$
\|f\|_{\dot{K}_{p(\cdot)}^{\alpha, q}(w)}:=\left(\sum_{k=-\infty}^{\infty} 2^{\alpha k q}\left\|f \chi_{k}\right\|_{L^{p(\cdot)}(w)}\right)^{1 / q}<\infty
$$

Herz spaces with variable exponent were initially defined by the first author $[11,12]$. The weighted case has been recently studied by the authors [22, 23].

\subsection{Weighted Banach function spaces}

We introduce Banach function space and state fundamental properties of it based on the book [26] by Bennett and Sharpley. We additionally show some properties of Banach function spaces in terms of the boundedness of the Hardy-Littlewood maximal operator. We will also consider the weighted case based on [27] by Karlovich and Spitkovsky. 
Definition 6 Let $\mathcal{M}$ be the set of all complex-valued measurable functions defined on $\mathbb{R}^{n}$, and $X$ a linear subspace of $\mathcal{M}$.

1. The space $X$ is said to be a Banach function space if there exists a functional $\|\cdot\|_{X}: \mathcal{M} \rightarrow[0, \infty]$ satisfying the following properties: Let $f, g, f_{j} \in \mathcal{M}(j=1,2, \ldots)$, then

(a) $f \in X$ holds if and only if $\|f\|_{X}<\infty$.

(b) Norm property:

i. Positivity: $\|f\|_{X} \geq 0$.

ii. Strict positivity: $\|f\|_{X}=0$ holds if and only if $f(x)=0$ for almost every $x \in \mathbb{R}^{n}$.

iii. Homogeneity: $\|\lambda f\|_{X}=|\lambda| \cdot\|f\|_{X}$ holds for all $\lambda \in \mathbb{C}$.

iv. Triangle inequality: $\|f+g\|_{X} \leq\|f\|_{X}+\|g\|_{X}$.

(c) Symmetry: $\|f\|_{X}=\||f|\|_{X}$.

(d) Lattice property: If $0 \leq g(x) \leq f(x)$ for almost every $x \in \mathbb{R}^{n}$, then $\|g\|_{X} \leq\|f\|_{X}$.

(e) Fatou property: If $0 \leq f_{j}(x) \leq f_{j+1}(x)$ for all $j$ and $f_{j}(x) \rightarrow f(x)$ as $j \rightarrow \infty$ for almost every $x \in \mathbb{R}^{n}$, then $\lim _{j \rightarrow \infty}\left\|f_{j}\right\|_{X}=\|f\|_{X}$.

(f) For every measurable set $F \subset \mathbb{R}^{n}$ such that $|F|<\infty,\left\|\chi_{F}\right\|_{X}$ is finite. Additionally there exists a constant $C_{F}>0$ depending only on $F$ so that $\int_{F}|h(x)| \mathrm{d} x \leq C_{F}\|h\|_{X}$ holds for all $h \in X$.

2. Suppose that $X$ is a Banach function space equipped with a norm $\|\cdot\|_{X}$. The associated space $X^{\prime}$ is defined by

$$
X^{\prime}:=\left\{f \in \mathcal{M}:\|f\|_{X^{\prime}}<\infty\right\}
$$

where

$$
\|f\|_{X^{\prime}}:=\sup _{g}\left\{\left|\int_{\mathbb{R}^{n}} f(x) g(x) \mathrm{d} x\right|:\|g\|_{X} \leq 1\right\}
$$

The proof of the following fundamental lemma is found in [26].

\section{Lemma 2 Let $X$ be a Banach function space. Then the following hold:}

1. The associated space $X^{\prime}$ is also a Banach function space.

2. (The Lorentz-Luxemburg theorem.) $\left(X^{\prime}\right)^{\prime}=X$ holds, in particular, the norms $\|\cdot\|_{\left(X^{\prime}\right)^{\prime}}$ and $\|\cdot\|_{X}$ are equivalent.

3. (The generalized Hölder inequality.) If $f \in X$ and $g \in X^{\prime}$, then we have

$$
\int_{\mathbb{R}^{n}}|f(x) g(x)| \mathrm{d} x \leq\|f\|_{X}\|g\|_{X^{\prime}}
$$

An easy application of the generalized Hölder inequality gives us the following lemma.

Lemma 3 If $X$ is a Banach function space, then we have that for all balls $B$,

$$
1 \leq \frac{1}{|B|}\left\|\chi_{B}\right\|_{X}\left\|\chi_{B}\right\|_{X^{\prime}}
$$


Kováčik and Rákosník [24] have proved that the generalized Lebesgue space $L^{p(\cdot)}\left(\mathbb{R}^{n}\right)$ with variable exponent $p(\cdot)$ is a Banach function space and the associate space is $L^{p^{\prime}(\cdot)}\left(\mathbb{R}^{n}\right)$ with norm equivalence.

If we assume some conditions for the boundedness of the Hardy-Littlewood maximal operator $M$ on $\mathrm{X}$, then the norm $\|\cdot\|_{X}$ has properties similar to the classical Muckenhoupt weights.

Lemma 4 Let $X$ be a Banach function space. Suppose that the Hardy-Littlewood maximal operator $M$ is weakly bounded on $X$, that is,

$$
\left\|\chi_{\{M f>\lambda\}}\right\|_{X} \lesssim \lambda^{-1}\|f\|_{X}
$$

is true for all $f \in X$ and all $\lambda>0$. Then we have

$$
\sup _{B: \text { ball }} \frac{1}{|B|}\left\|\chi_{B}\right\|_{X}\left\|\chi_{B}\right\|_{X^{\prime}}<\infty
$$

The proof of Lemma 4 is found in the first author's paper [19], Lemmas 2.4 and 2.5, and [28], Lemmas G' and $H$.

Remark 1 If $M$ is bounded on $X$, that is, $\|M f\|_{X} \lesssim\|f\|_{X}$ holds for all $f \in X$, then we can easily check that (6) holds. On the other hand, if $M$ is bounded on the associate space $X^{\prime}$, then Lemma 2 shows that (7) is true.

Below we define weighted Banach function space and give some of its property. Let $X$ be a Banach function space. The set $X_{\mathrm{loc}}\left(\mathbb{R}^{n}\right)$ consists of all measurable functions $f$ such that $f \chi_{E} \in X$ for any compact set $E$ with $|E|<\infty$. Given a function $W$ such that $0<W(x)<$ $\infty$ for almost every $x \in \mathbb{R}^{n}, W \in X_{\mathrm{loc}}\left(\mathbb{R}^{n}\right)$ and $W^{-1} \in\left(X^{\prime}\right)_{\text {loc }}\left(\mathbb{R}^{n}\right)$, we define the weighted Banach function space

$$
X\left(\mathbb{R}^{n}, W\right):=\{f \in \mathcal{M}: f W \in X\} .
$$

Then the following hold.

\section{Lemma 5}

1. The weighted Banach function space $X\left(\mathbb{R}^{n}, W\right)$ is a Banach function space equipped the norm

$$
\|f\|_{X\left(\mathbb{R}^{n}, W\right)}:=\|f W\|_{X}
$$

2. The associate space of $X\left(\mathbb{R}^{n}, W\right)$ is a Banach function space and equals $X^{\prime}\left(\mathbb{R}^{n}, W^{-1}\right)$.

The properties above naturally arise from those of the usual Banach function spaces and the proof is found in [27].

Remark 2 Let $p(\cdot) \in \mathcal{P}\left(\mathbb{R}^{n}\right)$. Comparing the definition of $X\left(\mathbb{R}^{n}, W\right)$ with weighted Lebesgue spaces $L^{p(\cdot)}\left(w^{p(\cdot)}\right)$ and $L^{p^{\prime}(\cdot)}\left(w^{-p^{\prime}(\cdot)}\right)$ respectively, we obtain the following:

1. If we take $X=L^{p(\cdot)}\left(\mathbb{R}^{n}\right)$ and $W=w$, then we have $L^{p(\cdot)}\left(\mathbb{R}^{n}, w\right)=L^{p(\cdot)}\left(w^{p(\cdot)}\right)$. 
2. If we take $X=L^{p^{\prime}(\cdot)}\left(\mathbb{R}^{n}\right)$ and $W=w^{-1}$, then we have $L^{p^{\prime}(\cdot)}\left(\mathbb{R}^{n}, w^{-1}\right)=L^{p^{\prime}(\cdot)}\left(w^{-p^{\prime}(\cdot)}\right)$.

Therefore Lemma 5 yields

$$
\left(L^{p(\cdot)}\left(w^{p(\cdot)}\right)\right)^{\prime}=\left(L^{p(\cdot)}\left(\mathbb{R}^{n}, w\right)\right)^{\prime}=L^{p^{\prime}(\cdot)}\left(\mathbb{R}^{n}, w^{-1}\right)=L^{p^{\prime}(\cdot)}\left(w^{-p^{\prime}(\cdot)}\right)
$$

The next lemma has been initially proved by the first author [12] in the case that $X=$ $L^{p(\cdot)}\left(\mathbb{R}^{n}\right)$, however, his argument depends on Diening's work [6]. Cruz-Uribe, Hernández and Martell [29] have recently given its alternative proof based on the Rubio de Francia algorithm [30-32]. As is mentioned in the authors' preprint [22], the proof due to [29] is self-contained and valid for general Banach function spaces $X$.

Lemma 6 Let $X$ be a Banach function space. Suppose that $M$ is bounded on the associate space $X^{\prime}$. Then there exists a constant $0<\delta<1$ such that for all balls $B \subset \mathbb{R}^{n}$ and all measurable sets $E \subset B$,

$$
\frac{\left\|\chi_{E}\right\|_{X}}{\left\|\chi_{B}\right\|_{X}} \lesssim\left(\frac{|E|}{|B|}\right)^{\delta}
$$

Proof For the reader's convenience we shall give the proof based on [29]. Let $A$ := $\|M\|_{X^{\prime} \rightarrow X^{\prime}}$ and define a function

$$
\operatorname{Rg}(x):=\sum_{k=0}^{\infty} \frac{M^{k} g(x)}{(2 A)^{k}} \quad\left(g \in X^{\prime}\right),
$$

where

$$
M^{k} g:= \begin{cases}|g| & (k=0), \\ M g & (k=1), \\ M\left(M^{k-1} g\right) & (k \geq 2) .\end{cases}
$$

For every $g \in X$, the function $\operatorname{Rg}$ satisfies the following properties:

1. $|g(x)| \leq R g(x)$ for almost every $x \in \mathbb{R}^{n}$.

2. $\|R g\|_{X^{\prime}} \leq 2\|g\|_{X^{\prime}}$, namely the operator $R$ is bounded on $X^{\prime}$.

3. $M(R g)(x) \leq 2 A R g(x)$, that is, $R g$ is a Muckenhoupt $A_{1}$ weight. We note that the constant $2 A$ appearing in the right-hand side is independent of $g$ and $x$.

We can write $\operatorname{Rg}(S)=\int_{S} \operatorname{Rg}(x) \mathrm{d} x$ for every measurable set $S \subset \mathbb{R}^{n}$ because $\operatorname{Rg}$ is a weight. Thus by virtue of [33], Chapter 7, we can take positive constants $C$ and $\delta<1$ depending only on $A$ and $n$ so that, for all balls $B$ and all measurable sets $E \subset B$,

$$
\frac{\operatorname{Rg}(E)}{\operatorname{Rg}(B)} \leq C\left(\frac{|E|}{|B|}\right)^{\delta}
$$

holds. Now we fix $g \in X^{\prime}$ with $\|g\|_{X^{\prime}} \leq 1$ arbitrarily. By virtue of generalized Hölder's inequality we have

$$
\begin{aligned}
\int_{\mathbb{R}^{n}}\left|\chi_{E}(x) g(x)\right| \mathrm{d} x & \leq \operatorname{Rg}(E) \leq C\left(\frac{|E|}{|B|}\right)^{\delta} \cdot \operatorname{Rg}(B) \leq C\left(\frac{|E|}{|B|}\right)^{\delta} \cdot\left\|\chi_{B}\right\|_{X}\|\operatorname{Rg}\|_{X^{\prime}} \\
& \lesssim\left(\frac{|E|}{|B|}\right)^{\delta}\left\|\chi_{B}\right\|_{X} .
\end{aligned}
$$


Therefore by the duality we get

$$
\left\|\chi_{E}\right\|_{X} \lesssim \sup _{g}\left\{\left|\int_{\mathbb{R}^{n}} \chi_{E}(x) g(x) \mathrm{d} x\right|: g \in X^{\prime},\|g\|_{X^{\prime}} \leq 1\right\} \lesssim\left(\frac{|E|}{|B|}\right)^{\delta}\left\|\chi_{B}\right\|_{X}
$$

This completes the proof of the lemma.

\section{The main results}

\subsection{Remarks on boundedness of fractional integrals on Lebesgue spaces}

Definition 7 Given $p(\cdot) \in \mathcal{P}\left(\mathbb{R}^{n}\right)$ and a weight $w$, we say $(p(\cdot), w)$ is an $M$-pair if the maximal operator $M$ is bounded on $L^{p(\cdot)}\left(w^{p(\cdot)}\right)$ and on $L^{p^{\prime}(\cdot)}\left(w^{-p^{\prime}(\cdot)}\right)$.

Let $0<\beta<n$. Then the fractional integral operator $I^{\beta}$ is defined by

$$
I^{\beta} f(x):=\int_{\mathbb{R}^{n}} \frac{f(y)}{|x-y|^{n-\beta}} \mathrm{d} y .
$$

Cruz-Uribe and Wang [21], Corollary 3.7, have obtained the following boundedness of $I^{\beta}$ on weighted variable exponent Lebesgue spaces.

Theorem 3 Let $p_{1}(\cdot) \in \mathcal{P}\left(\mathbb{R}^{n}\right) \cap L H\left(\mathbb{R}^{n}\right), 0<\beta<n / p_{1}^{+}$and $\sigma:=(n / \beta)^{\prime}$. Define $p_{2}(\cdot)$ by $1 / p_{2}(\cdot) \equiv 1 / p_{1}(\cdot)-\beta / n$. Then for all weights $w$ such that $\left(p_{2}(\cdot) / \sigma, w^{\sigma}\right)$ is an $M-p a i r, I^{\beta}$ is bounded from $L^{p_{1}(\cdot)}\left(w^{p_{1}(\cdot)}\right)$ to $L^{p_{2}(\cdot)}\left(w^{p_{2}(\cdot)}\right)$.

Let $w \in A\left(p_{1}(\cdot), p_{2}(\cdot)\right)$. Note that $p_{2}(\cdot) / \sigma=1+p_{2}(\cdot) / p_{1}^{\prime}(\cdot)$. Lemma 1 and the equivalence

$$
w^{p_{2}(\cdot)} \in A_{1+p_{2}(\cdot) / p_{1}^{\prime}(\cdot)}=A_{p_{2}(\cdot) / \sigma} \quad \Longleftrightarrow \quad w^{-\sigma\left(p_{2}(\cdot) / \sigma\right)^{\prime}} \in A_{\left(p_{2}(\cdot) / \sigma\right)^{\prime}}
$$

imply that $M$ is bounded on $L^{p_{2}(\cdot) / \sigma}\left(w^{\sigma \cdot p_{2}(\cdot) / \sigma}\right)$ and on $L^{\left(p_{2}(\cdot) / \sigma\right)^{\prime}}\left(w^{-\sigma\left(p_{2}(\cdot) / \sigma\right)^{\prime}}\right)$. Therefore, $\left(p_{2}(\cdot) / \sigma, w^{\sigma}\right)$ is an $M$-pair whenever

$$
w \in A\left(p_{1}(\cdot), p_{2}(\cdot)\right), \quad p_{1}(\cdot), p_{2}(\cdot) \in \mathcal{P}\left(\mathbb{R}^{n}\right) \cap L H\left(\mathbb{R}^{n}\right) \quad \text { and } \quad 0<\beta<n / p_{1}^{+} .
$$

Hence we have the following corollary.

Corollary 2 Let $p_{1}(\cdot) \in \mathcal{P}\left(\mathbb{R}^{n}\right) \cap L H\left(\mathbb{R}^{n}\right)$ and $0<\beta<n / p_{1}^{+}$. Define $p_{2}(\cdot)$ by $1 / p_{2}(\cdot) \equiv$ $1 / p_{1}(\cdot)-\beta / n$. If $w \in A\left(p_{1}(\cdot), p_{2}(\cdot)\right)$, then $I^{\beta}$ is bounded from $L^{p_{1}(\cdot)}\left(w^{p_{1}(\cdot)}\right)$ to $L^{p_{2}(\cdot)}\left(w^{p_{2}(\cdot)}\right)$.

\subsection{Boundedness of fractional integrals on Herz spaces}

Let $p_{2}(\cdot) \in \mathcal{P}\left(\mathbb{R}^{n}\right) \cap L H\left(\mathbb{R}^{n}\right)$ and $w^{p_{2}(\cdot)} \in A_{1}$. Then the monotone property yields $w^{p_{2}(\cdot)} \in$ $A_{p_{2}(\cdot)}$. Hence the Hardy-Littlewood maximal operator $M$ is bounded on $L^{p_{2}(\cdot)}\left(w^{p_{2}(\cdot)}\right)$. On the other hand, by the definition of $A_{p(\cdot)}$ it is easy to see that $w^{p_{2}(\cdot)} \in A_{p_{2}(\cdot)}$ implies $w^{-p_{2}^{\prime}(\cdot)} \in$ $A_{p_{2}^{\prime}(\cdot)}$. Thus $M$ is bounded on $L^{p_{2}^{\prime}(\cdot)}\left(w^{-p_{2}^{\prime}(\cdot)}\right)$. Therefore applying Remark 2, Lemma 6, and the Lorentz-Luxemburg theorem we can take constants $\delta_{1}, \delta_{2} \in(0,1)$ such that

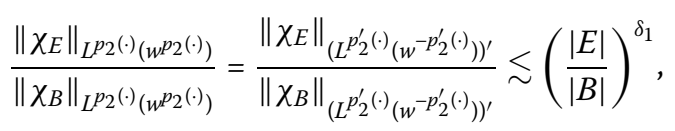




$$
\frac{\left\|\chi_{E}\right\|_{\left(L ^ { p _ { 2 } ( \cdot ) } \left(w^{\left.\left.p_{2}(\cdot)\right)\right)^{\prime}}\right.\right.}}{\left\|\chi_{B}\right\|_{\left(L^{p_{2}(\cdot)}\left(w^{p_{2}(\cdot)}\right)\right)^{\prime}}} \lesssim\left(\frac{|E|}{|B|}\right)^{\delta_{2}}
$$

for all balls $B$ and all measurable sets $E \subset B$. Now we take a positive number $\beta$ so that $0<\beta<n\left(\delta_{1}+\delta_{2}\right)$. Then we can take a real number $\alpha$ such that $-n \delta_{1}<\alpha<n \delta_{2}-\beta$ because the choice of $\beta$ shows $-n \delta_{1}<n \delta_{2}-\beta$. Using $p_{2}(\cdot)$ and $\beta$, we additionally define $p_{1}(\cdot)$ so that $1 / p_{2}(\cdot) \equiv 1 / p_{1}(\cdot)-\beta / n$ holds. We see that $p_{1}(\cdot)$ satisfies $p_{1}(\cdot) \in \mathcal{P}\left(\mathbb{R}^{n}\right) \cap L H\left(\mathbb{R}^{n}\right)$ and $0<\beta<n / p_{1}^{+}$. Applying the monotone property again we have $w^{p_{2}(\cdot)} \in A_{1} \subset A_{1+p_{2}(\cdot) / p_{1}^{\prime}(\cdot)}$. Thus by Lemma 1 we get $w \in A\left(p_{1}(\cdot), p_{2}(\cdot)\right)$.

Therefore in the setting as above we can apply Corollary 2 to obtain the boundedness of the fractional integral operator $I^{\beta}$ on Herz spaces.

Theorem 4 Let $0<q_{1} \leq q_{2}<\infty, p_{2}(\cdot) \in \mathcal{P}\left(\mathbb{R}^{n}\right) \cap L H\left(\mathbb{R}^{n}\right)$, $w^{p_{2}(\cdot)} \in A_{1}, \delta_{1}, \delta_{2} \in(0,1)$ be the constants appearing in (9) and (10) respectively, $0<\beta<n\left(\delta_{1}+\delta_{2}\right)$ and $-n \delta_{1}<\alpha<n \delta_{2}-\beta$. Define $p_{1}(\cdot)$ by $1 / p_{2}(\cdot) \equiv 1 / p_{1}(\cdot)-\beta / n$. Then the fractional integral operator $I^{\beta}$ is a bounded operator from $\dot{K}_{p_{2}(\cdot)}^{\alpha, q_{2}}\left(w^{p_{2}(\cdot)}\right)$ to $\dot{K}_{p_{1}(\cdot)}^{\alpha, q_{1}}\left(w^{p_{1}(\cdot)}\right)$.

Proof Let $f \in \dot{K}_{p_{1}(\cdot)}^{\alpha, q_{1}}\left(w^{p_{1}(\cdot)}\right)$. Then, by the Jensen inequality, we have

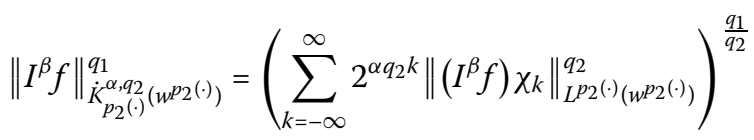

$$
\begin{aligned}
& \leq \sum_{k=-\infty}^{\infty} 2^{\alpha q_{1} k}\left\|\left(I^{\beta} f\right) \chi_{k}\right\|_{L^{p_{2}(\cdot)}\left(w^{p_{2}(\cdot)}\right)}^{q_{1}} .
\end{aligned}
$$

Let $f_{j}:=f \chi_{j}$ for any $j \in \mathbb{Z}$. Then $f=\sum_{j=-\infty}^{\infty} f_{j}$. So we have

$$
\begin{aligned}
& \left\|I^{\beta} f\right\|_{\dot{K}_{p_{2}(\cdot)}^{\alpha, q_{2}\left(w_{2}(\cdot)\right)}}^{q_{1}} \leq \sum_{k=-\infty}^{\infty} 2^{\alpha q_{1} k}\left\|\left(I^{\beta} f\right) \chi_{k}\right\|_{L^{p_{2}(\cdot)}\left(w^{p_{2}(\cdot)}\right)}^{q_{1}} \\
& \leq \sum_{k=-\infty}^{\infty} 2^{\alpha q_{1} k}\left(\sum_{j \leq k-2}\left\|\left(I^{\beta} f_{j}\right) \chi_{k}\right\|_{L^{p_{2}(\cdot)}\left(w^{p_{2}(\cdot)}\right)}\right)^{q_{1}} \\
& +\sum_{k=-\infty}^{\infty} 2^{\alpha q_{1} k}\left(\sum_{j=k-1}^{k+1}\left\|\left(I^{\beta} f_{j}\right) \chi_{k}\right\|_{L^{p_{2}(\cdot)}\left(w^{p_{2}(\cdot)}\right)}\right)^{q_{1}}
\end{aligned}
$$

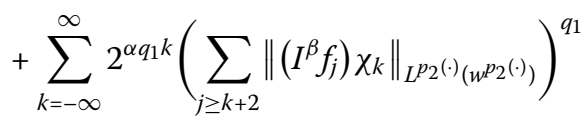

$$
\begin{aligned}
& =: U_{1}+U_{2}+U_{3} \text {. }
\end{aligned}
$$

Step 1 . We estimate $U_{1}$. By the definition of $I^{\beta}$ and the generalized Hölder inequality, we obtain

$$
\begin{aligned}
\left|\left(I^{\beta} f_{j}\right)(x)\right| \chi_{k}(x) & \lesssim \chi_{k}(x) \int_{\mathbb{R}^{n}}|x-y|^{\beta-n}\left|f_{j}(y)\right| \mathrm{d} y \\
& \lesssim 2^{k(\beta-n)}\left\|f_{j}\right\|_{L^{p_{1}(\cdot)}\left(w^{\left.p_{1}(\cdot)\right)}\right)}\left\|\chi_{j}\right\|_{\left(L ^ { p _ { 1 } ( \cdot ) } \left(w^{\left.\left.p_{1}(\cdot)\right)\right)^{\prime}}\right.\right.} \chi_{k}(x) .
\end{aligned}
$$


By taking the $L^{p_{2}(\cdot)}\left(w^{p_{2}(\cdot)}\right)$-norm and using Lemma 4 , we have

$$
\begin{aligned}
& \left\|\left(I^{\beta} f_{j}\right) \chi_{k}\right\|_{L^{p_{2}(\cdot)}\left(w^{\left.p_{2}(\cdot)\right)}\right.} \\
& \lesssim 2^{k(\beta-n)}\left\|f_{j}\right\|_{L^{p_{1}(\cdot)}\left(w^{\left.p_{1}(\cdot)\right)}\right.}\left\|\chi_{j}\right\|_{\left(L^{p_{1}(\cdot)}\left(w^{p_{1}(\cdot)}\right)\right)^{\prime}}\left\|\chi_{B_{k}}\right\|_{L^{p_{2}(\cdot)\left(w^{p_{2}}(\cdot)\right)}}
\end{aligned}
$$

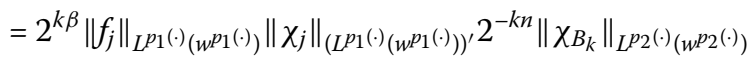

$$
\begin{aligned}
& \lesssim 2^{k \beta}\left\|f_{j}\right\|_{L^{p_{1}(\cdot)}\left(w^{p_{1}(\cdot)}\right)}\left\|\chi_{j}\right\|_{\left(L^{p_{1}(\cdot)}\left(w^{p_{1}(\cdot)}\right)\right)^{\prime}}\left\|\chi_{B_{k}}\right\|_{\left(L^{p_{2}(\cdot)}\left(w^{p_{2}(\cdot)}\right)\right)^{\prime}}^{-} \text {. }
\end{aligned}
$$

By (10), we see that

$$
\begin{aligned}
& \left\|\left(I^{\beta} f_{j}\right) \chi_{k}\right\|_{L^{p_{2}(\cdot)}\left(w^{p_{2}(\cdot)}\right)} \\
& \left.\lesssim 2^{k \beta}\left\|f_{j}\right\|_{L^{p_{1}(\cdot)}\left(w^{p_{1}(\cdot)}\right)}\left\|\chi_{j}\right\|_{\left(L ^ { p _ { 1 } ( \cdot ) } \left(w^{\left.\left.p^{1} \cdot(\cdot)\right)\right)^{\prime}}\right.\right.}\left\|\chi_{B_{k}}\right\|_{L^{\left(p_{2}(\cdot)\right.}}^{-1}\left(w^{p_{2}(\cdot)}\right)\right)^{\prime}
\end{aligned}
$$

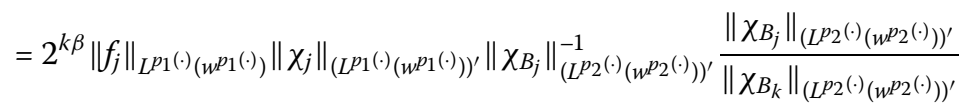

$$
\begin{aligned}
& \lesssim 2^{k \beta} 2^{n \delta_{2}(j-k)}\left\|f_{j}\right\|_{L^{p_{1}(\cdot)}\left(w^{p_{1}(\cdot)}\right)}\left\|\chi_{j}\right\|_{\left(L^{p_{1}(\cdot)}\left(w^{p_{1}(\cdot)}\right)\right)^{\prime}}\left\|\chi_{B_{j}}\right\|_{\left(L ^ { p _ { 2 } ( \cdot ) } \left(w^{\left.\left.p_{2}(\cdot)\right)\right)^{\prime}}\right.\right.}^{-1} .
\end{aligned}
$$

By the obvious inequality $2^{j \beta} \chi_{B_{j}}(x) \lesssim\left(I^{\beta} f_{B_{j}}\right)(x)$ and the boundedness of $I^{\beta}: L^{p_{1}(\cdot)}\left(w^{p_{1}(\cdot)}\right) \rightarrow$ $L^{p_{2}(\cdot)}\left(w^{p_{2}(\cdot)}\right)$, we have

$$
\left\|\chi_{B_{j}}\right\|_{L^{p_{2}(\cdot)}\left(w^{p_{2}(\cdot)}\right)} \lesssim 2^{-j \beta}\left\|I^{\beta} \chi_{B_{j}}\right\|_{L^{p_{2}(\cdot)}\left(w^{p_{2}(\cdot)}\right)} \lesssim 2^{-j \beta}\left\|\chi_{B_{j}}\right\|_{L^{p_{1}(\cdot)}\left(w^{p_{1}(\cdot)}\right)} .
$$

By using Lemma 4 again, we obtain

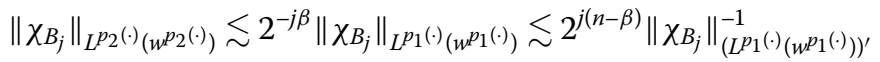

$$
\begin{aligned}
& \lesssim 2^{j(n-\beta)}\left\|\chi_{j}\right\|_{\left(L^{p_{1}(\cdot)}\left(w^{p_{1}(\cdot)}\right)\right)^{\circ}} .
\end{aligned}
$$

Combining (13) and (14) and using Lemma 3, we get

$$
\begin{aligned}
& \left\|\left(I^{\beta} f_{j}\right) \chi_{k}\right\|_{L^{p_{2}(\cdot)}\left(w^{\left.p_{2}(\cdot)\right)}\right.}
\end{aligned}
$$

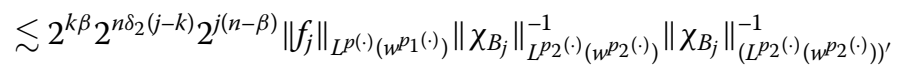

$$
\begin{aligned}
& =2^{\left(\beta-n \delta_{2}\right)(k-j)}\left\|f_{j}\right\|_{L^{p_{1}(\cdot)}\left(w^{\left.p_{1}(\cdot)\right)}\right.}\left(2^{-j n}\left\|\chi_{B_{j}}\right\|_{L^{p_{2}(\cdot)}\left(w^{p_{2}(\cdot)}\right)}\left\|\chi_{B_{j}}\right\|_{\left(L^{p_{2}(\cdot)}\left(w^{p_{2}(\cdot)}\right)\right)^{\prime}}\right)^{-1} \\
& \lesssim 2^{\left(\beta-n \delta_{2}\right)(k-j)}\left\|f_{j}\right\|_{L^{p_{1}(\cdot)}\left(w^{p_{1}(\cdot)}\right)} \text {. }
\end{aligned}
$$

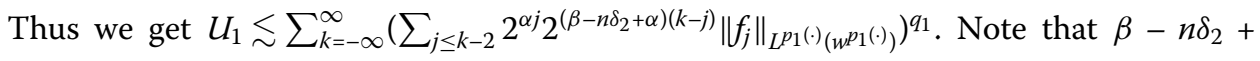
$\alpha<0$. We consider the two cases ' $1<q_{1}<\infty$ ' and ' $0<q_{1} \leq 1$ '.

If $1<q_{1}<\infty$, then, by using the Hölder inequality, we obtain

$$
\begin{aligned}
U_{1} & \lesssim \sum_{k=-\infty}^{\infty}\left(\sum_{j \leq k-2} 2^{\alpha j} 2^{\left(\beta-n \delta_{2}+\alpha\right)(k-j)}\left\|f_{j}\right\|_{L^{p_{1}(\cdot)}\left(w^{\left.p_{1}(\cdot)\right)}\right)}\right)^{q_{1}} \\
& \lesssim \sum_{k=-\infty}^{\infty}\left(\sum_{j \leq k-2} 2^{\alpha j q_{1}} 2^{\left(\beta-n \delta_{2}+\alpha\right)(k-j) q_{1} / 2}\left\|f_{j}\right\|_{L^{p_{1}(\cdot)}\left(w^{\left.p_{1}(\cdot)\right)}\right)}^{q_{1}}\right)\left(\sum_{j \leq k-2} 2^{\left(\beta-n \delta_{2}+\alpha\right)(k-j) q_{1}^{\prime} / 2}\right)^{q_{1} / q_{1}^{\prime}}
\end{aligned}
$$




$$
\begin{aligned}
& \lesssim \sum_{k=-\infty}^{\infty} \sum_{j \leq k-2} 2^{\alpha j q_{1}} 2^{\left(\beta-n \delta_{2}+\alpha\right)(k-j) q_{1} / 2}\left\|f_{j}\right\|_{L^{p_{1}(\cdot)}\left(w^{p_{1}(\cdot)}\right)}^{q_{1}} \\
& =\sum_{j=-\infty}^{\infty} 2^{\alpha j q_{1}}\left\|f_{j}\right\|_{L^{p_{1}(\cdot)}\left(w^{p_{1}(\cdot)}\right.}^{q_{1}} \sum_{k \leq j-2} 2^{\left(\beta-n \delta_{2}+\alpha\right)(k-j) q_{1} / 2} \\
& \lesssim\|f\|_{\dot{K}_{p_{1}(\cdot)}^{\alpha, q_{1}}\left(w^{p_{1}(\cdot)}\right)^{\cdot}}^{q_{1}}
\end{aligned}
$$

If $0<q_{1} \leq 1$, then by using the Jensen inequality we obtain

$$
\begin{aligned}
U_{1} & \lesssim \sum_{k=-\infty}^{\infty}\left(\sum_{j \leq k-2} 2^{\alpha j} 2^{\left(\beta-n \delta_{2}+\alpha\right)(k-j)}\left\|f_{j}\right\|_{L^{p_{1}(\cdot)}\left(w^{\left.p_{1}(\cdot)\right)}\right)}\right)^{q_{1}} \\
& \lesssim \sum_{k=-\infty}^{\infty} \sum_{j \leq k-2} 2^{\alpha j q_{1}} 2^{\left(\beta-n \delta_{2}+\alpha\right)(k-j) q_{1}}\left\|f_{j}\right\|_{L^{p_{1}(\cdot)}\left(w^{p_{1}(\cdot)}\right)}^{q_{1}} \\
& =\sum_{j=-\infty}^{\infty} 2^{\alpha j q_{1}}\left\|f_{j}\right\|_{L^{p_{1}(\cdot)}\left(w^{\left.p_{1}(\cdot)\right)}\right.}^{q_{1}} \sum_{k \leq j-2} 2^{\left(\beta-n \delta_{2}+\alpha\right)(k-j) q_{1}} \\
& \lesssim\|f\|_{\dot{K}_{p_{1}(\cdot)}^{\alpha, q_{1}}}^{\left.q_{1} w_{1}(\cdot)\right)^{\prime}}
\end{aligned}
$$

Step 2. We estimate $U_{2}$. Using Corollary 2 and $-1 \leq k-j \leq 1$, it is easy to see that

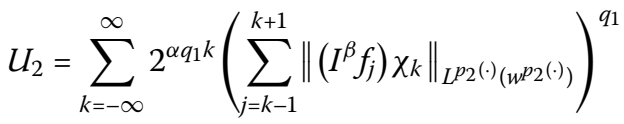

$$
\begin{aligned}
& =\sum_{k=-\infty}^{\infty}\left(\sum_{j=k-1}^{k+1} 2^{\alpha(k-j)} 2^{\alpha j}\left\|f_{j}\right\|_{L^{p_{1}(\cdot)}\left(w^{\left.p_{1}(\cdot)\right)}\right)}\right)^{q_{1}}
\end{aligned}
$$

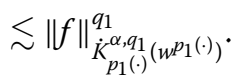

Step 3 . We estimate $U_{3}$. Using the generalized Hölder inequality, we have, for every $j, k \in$ $\mathbb{Z}$ with $j \geq k+2$,

$$
\begin{aligned}
\left|I^{\beta}\left(f_{j}\right) \chi_{k}(x)\right| & \lesssim 2^{j(-n+\beta)} \int_{B_{j}}|f(y)| \mathrm{d} y \cdot \chi_{k}(x) \\
& \lesssim 2^{j(-n+\beta)}\|f w\|_{L^{p_{1}(\cdot)}}\left\|\chi_{j}\right\|_{L^{p^{\prime}(\cdot)}\left(w^{\left.-p_{1}^{\prime}(\cdot)\right)}\right.} \chi_{k}(x) .
\end{aligned}
$$

By taking the $L^{p_{2}(\cdot)}\left(w^{p_{2}(\cdot)}\right)$-norm and (9), we have

$$
\begin{aligned}
& \left\|\left(I^{\beta} f_{j}\right) \chi_{k}\right\|_{L^{p_{2}(\cdot)}\left(w^{p_{2}(\cdot)}\right)} \\
& \lesssim 2^{j(-n+\beta)}\left\|f_{j} w\right\|_{L^{p_{1}(\cdot)}}\left\|\chi_{j}\right\|_{L^{p_{1}^{\prime}(\cdot)}\left(w^{-p_{1}^{\prime}(\cdot)}\right)}\left\|\chi_{k}\right\|_{L^{p_{2}(\cdot)}\left(w^{\left.p_{2}(\cdot)\right)}\right.} \\
& \lesssim 2^{j(-n+\beta)}\left\|f_{j} w\right\|_{L^{p_{1}(\cdot)}}\left\|\chi_{j}\right\|_{L^{p_{1}^{(\cdot)}\left(w^{-}-p_{1}^{\prime}(\cdot)\right)}}\left\|\chi_{j}\right\|_{L^{p_{2}(\cdot)}\left(w^{p_{2}(\cdot)}\right)} \frac{\left\|\chi_{k}\right\|_{L^{p_{2}(\cdot)}\left(w^{p_{2}(\cdot)}\right)}}{\left\|\chi_{j}\right\|_{L^{p_{2}(\cdot)}\left(w^{p_{2}(\cdot)}\right)}} \\
& \lesssim 2^{j(-n+\beta)} 2^{n \delta_{1}(k-j)}\left\|f_{j} w\right\|_{L^{p_{1}(\cdot)}}\left\|\chi_{j}\right\|_{L^{p_{1}^{\prime}(\cdot)}\left(w^{-p_{1}^{\prime} \cdot(\cdot)}\right)}\left\|\chi_{j}\right\|_{L^{p_{2}(\cdot)}\left(w^{p_{2}(\cdot)}\right)} \text {. }
\end{aligned}
$$


By the definition of $A\left(p_{1}(\cdot), p_{2}(\cdot)\right)$, we obtain

$$
\begin{aligned}
&\left\|\chi_{j}\right\|_{L^{p_{1}^{\prime}(\cdot)}\left(w^{-p_{1}^{\prime}(\cdot)}\right)}\left\|\chi_{j}\right\|_{L^{p_{2}(\cdot)}\left(w^{\left.p_{2}(\cdot)\right)}\right.} \lesssim\left\|\chi_{B_{j}}\right\|_{L^{p_{1}^{\prime}(\cdot)}\left(w^{-p_{1}^{\prime} \cdot(\cdot)}\right)}\left\|\chi_{B_{j}}\right\|_{L^{p_{2}(\cdot)}\left(w^{\left.p_{2}(\cdot)\right)}\right.} \\
& \lesssim\left\|w^{-1} \chi_{B_{j}}\right\|_{L^{p_{1}^{\prime}(\cdot)}}\left\|w \chi_{B_{j}}\right\|_{L^{p_{2}(\cdot)}} \\
& \lesssim 2^{j n(1-\beta / n)}
\end{aligned}
$$

Hence we have

$$
\begin{aligned}
& \left\|\left(I^{\beta} f_{j}\right) \chi_{k}\right\|_{L^{p_{2}(\cdot)}\left(w^{p_{2}(\cdot)}\right)} \\
& \quad \lesssim 2^{j(-n+\beta)} 2^{n \delta_{1}(k-j)}\left\|f_{j} w\right\|_{L^{p_{1}(\cdot)}}\left\|\chi_{j}\right\|_{L^{p_{1}^{\prime}(\cdot)}\left(w^{-p_{1}^{p_{1}} \cdot(\cdot)}\right)}\left\|\chi_{j}\right\|_{L^{p_{2}(\cdot)}\left(w^{\left.p_{2}(\cdot)\right)}\right.} \\
& \quad \lesssim 2^{j(-n+\beta)} 2^{n \delta_{1}(k-j)} 2^{j n(1-\beta / n)}\left\|f_{j} w\right\|_{L^{p_{1}(\cdot)}} \\
& \quad=2^{n \delta_{1}(k-j)}\left\|f_{j} w\right\|_{L^{p_{1}}(\cdot)} .
\end{aligned}
$$

Therefore we see that

$$
\begin{aligned}
U_{3} & =\sum_{k=-\infty}^{\infty} 2^{\alpha q_{1} k}\left(\sum_{j \geq k+2}\left\|\left(I^{\beta} f_{j}\right) \chi_{k}\right\|_{L^{p_{2}(\cdot)}\left(w^{\left.p_{2}(\cdot)\right)}\right)}\right)^{q_{1}} \\
& \lesssim \sum_{k=-\infty}^{\infty} 2^{\alpha q_{1} k}\left(\sum_{j \geq k+2} 2^{n \delta_{1}(k-j)}\left\|f_{j} w\right\|_{L^{p_{1}(\cdot)}}\right)^{q_{1}} \\
& \lesssim \sum_{k=-\infty}^{\infty}\left(\sum_{j \geq k+2} 2^{\left(\alpha+n \delta_{1}\right)(k-j)} 2^{\alpha j}\left\|f_{j} w\right\|_{L^{p_{1}(\cdot)}}\right)^{q_{1}} .
\end{aligned}
$$

Note that $\alpha+n \delta_{1}>0$. We consider the two cases: ' $1<q_{1}<\infty$ ' and ' $0<q_{1} \leq 1$ '.

If $1<q_{1}<\infty$, then by using the Hölder inequality we obtain

$$
\begin{aligned}
& U_{3} \lesssim \sum_{k=-\infty}^{\infty}\left(\sum_{j \geq k+2} 2^{\left(\alpha+n \delta_{1}\right)(k-j)} 2^{\alpha j}\left\|f_{j} w\right\|_{L^{p_{1}(\cdot)}}\right)^{q_{1}} \\
& \lesssim \sum_{k=-\infty}^{\infty}\left(\sum_{j \geq k+2} 2^{\left(\alpha+n \delta_{1}\right)(k-j) q_{1} / 2} 2^{\alpha q_{1} j}\left\|f_{j} w\right\|_{L^{p_{1}(\cdot)}}^{q_{1}}\right)\left(\sum_{j \geq k+2} 2^{\left(\alpha+n \delta_{1}\right)(k-j) q_{1}^{\prime} / 2}\right)^{q_{1} / q_{1}^{\prime}} \\
& \lesssim \sum_{j=-\infty}^{\infty} 2^{\alpha q_{1} j}\left\|f_{j} w\right\|_{L^{p_{1}(\cdot)}}^{q_{1}} \sum_{k \leq j-2} 2^{\left(\alpha+n \delta_{1}\right)(k-j) q_{1} / 2} \\
& \lesssim\|f\|_{\dot{K}_{p_{1}(\cdot)}^{\alpha, q_{1}}\left(w^{p_{1}(\cdot)}\right)}^{q_{1}} \cdot
\end{aligned}
$$

If $0<q_{1} \leq 1$, then by using the Jensen inequality we obtain

$$
\begin{aligned}
U_{3} & \lesssim \sum_{k=-\infty}^{\infty}\left(\sum_{j \geq k+2} 2^{\left(\alpha+n \delta_{1}\right)(k-j)} 2^{\alpha j}\left\|f_{j} w\right\|_{L^{p_{1}(\cdot)}}\right)^{q_{1}} \\
& \lesssim \sum_{k=-\infty}^{\infty} \sum_{j \geq k+2} 2^{\left(\alpha+n \delta_{1}\right)(k-j) q_{1}} 2^{\alpha q_{1} j}\left\|f_{j} w\right\|_{L^{p_{1}(\cdot)}}^{q_{1}}
\end{aligned}
$$




$$
\begin{aligned}
& \lesssim \sum_{j=-\infty}^{\infty} 2^{\alpha q_{1} j}\left\|f_{j} w\right\|_{L^{p_{1}(\cdot)}}^{q_{1}} \sum_{k \leq j-2} 2^{\left(\alpha+n \delta_{1}\right)(k-j) q_{1}} \\
& \lesssim\|f\|_{\dot{K}_{p_{1}(\cdot)}^{\alpha, q_{1}}\left(w^{p_{1}(\cdot)}\right)^{\cdot}}^{q_{1}}
\end{aligned}
$$

Consequently we have proved the theorem.

\subsection{The non-homogeneous case}

In this paper we have defined the weighted Herz space with variable exponent and proved the boundedness of the fractional integrals on the spaces in the homogeneous case. Our argument is also valid for the non-homogeneous case.

Definition 8 Let $p(\cdot) \in \mathcal{P}\left(\mathbb{R}^{n}\right), 0<q<\infty$, and $\alpha \in \mathbb{R}$. For non-negative integer $k$, let

$$
C_{k}:= \begin{cases}R_{k} & (k \geq 1), \\ B_{0} & (k=0) .\end{cases}
$$

The non-homogeneous weighted Herz space $K_{p(\cdot)}^{\alpha, q}(w)$ is the collection of $f \in L_{\text {loc }}^{p(\cdot)}\left(\mathbb{R}^{n}, w\right)$ such that

$$
\|f\|_{K_{p(\cdot)}^{\alpha, q}(w)}:=\left(\sum_{k=0}^{\infty} 2^{\alpha k q}\left\|f \chi_{C_{k}}\right\|_{L^{p(\cdot)}(w)}\right)^{1 / q}<\infty .
$$

Theorem 5 Let $0<q_{1} \leq q_{2}<\infty, p_{2}(\cdot) \in \mathcal{P}\left(\mathbb{R}^{n}\right) \cap L H\left(\mathbb{R}^{n}\right)$, w $w^{p_{2}(\cdot)} \in A_{1}, \delta_{1}, \delta_{2} \in(0,1)$ be the constants appearing in (9) and (10) respectively, $0<\beta<n\left(\delta_{1}+\delta_{2}\right)$, and $-n \delta_{1}<\alpha<n \delta_{2}-\beta$. Define $p_{1}(\cdot)$ by $1 / p_{2}(\cdot) \equiv 1 / p_{1}(\cdot)-\beta / n$. Then the fractional integral operator $I^{\beta}$ is a bounded operator from $K_{p_{2}(\cdot)}^{\alpha, q_{2}}\left(w^{p_{2}(\cdot)}\right)$ to $K_{p_{1}(\cdot)}^{\alpha, q_{1}}\left(w^{p_{1}(\cdot)}\right)$.

Remark 3 In the main theorems we have assumed that $w^{p_{2}(\cdot)} \in A_{1}$ to obtain the boundedness of $I^{\beta}$. One may think that the condition $w^{p_{2}(\cdot)} \in A_{1}$ is too strong. In this paper we could not prove the main theorems under weaker conditions on $w$.

\section{Competing interests}

The authors declare that they have no competing interests.

Authors' contributions

The authors contributed equally to this work. All authors read and approved the final manuscript.

\section{Author details}

'Faculty of Education, Okayama University, 3-1-1 Tsushima-naka, Okayama, 700-8530, Japan. ${ }^{2}$ Department of Mathematics and Information Science, Tokyo Metropolitan University, Hachioji, 192-0397, Japan.

\footnotetext{
Acknowledgements

The first author was partially supported by Grand-in-Aid for Scientific Research (C), No. 15K04928, for Japan Society for the Promotion of Science. The second author was partially supported by Grand-in-Aid for Scientific Research (C), No. 16K05212, for Japan Society for the Promotion of Science. The authors are thankful to the referees, whose comments have improved the manuscript.
}

Received: 3 April 2016 Accepted: 5 August 2016 Published online: 16 August 2016

\section{References}

1. Capone, C, Cruz-Uribe, D, SFO, Fiorenza, A: The fractional maximal operator and fractional integrals on variable $L^{p}$ spaces. Rev. Mat. Iberoam. 23(3), 743-770 (2007) 
2. Cruz-Uribe, DV, Fiorenza, A: Variable Lebesgue Spaces. Foundations and Harmonic Analysis. Applied and Numerical Harmonic Analysis. Springer, Heidelberg (2013)

3. Cruz-Uribe, D, Fiorenza, A, Neugebauer, CJ: The maximal function on variable $L^{p}$ spaces. Ann. Acad. Sci. Fenn., Math. 28(1), 223-238 (2003)

4. Cruz-Uribe, D, Fiorenza, A, Neugebauer, CJ: Corrections to: 'The maximal function on variable $L^{p}$ spaces' $[$ Ann. Acad. Sci. Fenn. Math. 28 (2003), 223-238]. Ann. Acad. Sci. Fenn., Math. 29(1), 247-249 (2004)

5. Diening, L: Maximal function on generalized Lebesgue spaces $L^{p(\cdot)}$. Math. Inequal. Appl. 7(2), 245-253 (2004)

6. Diening, L: Maximal function on Musielak-Orlicz spaces and generalized Lebesgue spaces. Bull. Sci. Math. 129(8), 657-700 (2005)

7. Diening, L, Harjulehto, P, Hästö, P, Růžička, M: Lebesgue and Sobolev Spaces with Variable Exponents. Lecture Notes in Mathematics, vol. 2017. Springer, Heidelberg (2011)

8. Izuki, M, Nakai, E, Sawano, Y: Function spaces with variable exponents -an introduction-. Sci. Math. Jpn. 77(2), 187-315 (2014)

9. Cruz-Uribe, D, Fiorenza, A, Martell, JM, Pérez, C: The boundedness of classical operators on variable $L^{p}$ spaces. Ann. Acad. Sci. Fenn., Math. 31(1), 239-264 (2006)

10. Lu, SZ, Yang, DC: Hardy-Littlewood-Sobolev theorems of fractional integration on Herz-type spaces and its applications. Can. J. Math. 48(2), 363-380 (1996)

11. Izuki, M: Herz and amalgam spaces with variable exponent, the Haar wavelets and greediness of the wavelet system. East J. Approx. 15(1), 87-109 (2009)

12. Izuki, M: Boundedness of sublinear operators on Herz spaces with variable exponent and application to wavelet characterization. Anal. Math. 36(1), 33-50 (2010)

13. Izuki, M: Fractional integrals on Herz-Morrey spaces with variable exponent. Hiroshima Math. J. 40(3), 343-355 (2010)

14. Almeida, A, Drihem, D: Maximal, potential and singular type operators on Herz spaces with variable exponents. J. Math. Anal. Appl. 394(2), 781-795 (2012)

15. Muckenhoupt, B: Weighted norm inequalities for the Hardy maximal function. Trans. Am. Math. Soc. 165, 207-226 (1972)

16. Cruz-Uribe, D, Diening, L, Hästö, P: The maximal operator on weighted variable Lebesgue spaces. Fract. Calc. Appl. Anal. 14(3), 361-374 (2011)

17. Cruz-Uribe, D, Fiorenza, A, Neugebauer, CJ: Weighted norm inequalities for the maximal operator on variable Lebesgue spaces. J. Math. Anal. Appl. 394(2), 744-760 (2012)

18. Diening, L, Hästö, P: Muckenhoupt weights in variable exponent spaces. Preprint, available at http://www.helsinki.fi/ hasto/pp/p75_submit.pdf

19. Izuki, M: Remarks on Muckenhoupt weights with variable exponent. J. Anal. Appl. 11(1-2), $27-41$ (2013)

20. Izuki, M, Nakai, E, Sawano, Y: Wavelet characterization and modular inequalities for weighted Lebesgue spaces with variable exponent. Ann. Acad. Sci. Fenn., Math. 40(2), 551-571 (2015)

21. Cruz-Uribe, D, Wang, L-AD: Extrapolation and weighted norm inequalities in the variable Lebesgue spaces. Preprint. arXiv: 1408.4499

22. Izuki, M, Noi, T: An intrinsic square function on weighted Herz spaces with variable exponent. Preprint

23. Izuki, M, Noi, T: The Hardy-Littlewood maximal operator on some critical weighted Herz spaces with variable exponent. Preprint

24. Kováčik, O, Rákosník, J: On spaces $L^{p(x)}$ and $W^{k, p(x)}$. Czechoslov. Math. J. 41(4), 592-618 (1991)

25. Komori, Y, Matsuoka, K: Boundedness of several operators on weighted Herz spaces. J. Funct. Spaces Appl. 7(1), 1-12 (2009)

26. Bennett, C, Sharpley, R: Interpolation of Operators. Academic Press, Boston (1988)

27. Karlovich, AY, Spitkovsky, IM: The Cauchy singular integral operator on weighted variable Lebesgue spaces. In: Concrete Operators, Spectral Theory, Operators in Harmonic Analysis and Approximation. Oper. Theory Adv. Appl., vol. 236, pp. 275-291. Springer, Basel (2014)

28. Izuki, M, Sawano, Y, Tsutsui, Y: Variable Lebesgue norm estimates for BMO functions. II. Anal. Math. 40(3), 215-230 (2014)

29. Cruz-Uribe, D, Hernández, E, Martell, JM: Greedy bases in variable Lebesgue spaces. Monatshefte Math. 179(3), 355-378 (2016)

30. Rubio de Francia, JL: Factorization and extrapolation of weights. Bull. Am. Math. Soc. (N.S.) 7(2), 393-395 (1982)

31. Rubio de Francia, JL: A new technique in the theory of $A_{p}$ theory. In: Topics in Modern Harmonic Analysis, vol. I, II, Turin/Milan, 1982, pp. 571-579. Ist. Naz. Alta Mat. Francesco Severi, Rome (1983)

32. Rubio de Francia, JL: Factorization theory and $A_{p}$ weights. Am. J. Math. 106(3), 533-547 (1984)

33. Duoandikoetxea, J: Fourier Analysis. Graduate Studies in Math., vol. 29. Am. Math. Soc., Providence (2001) 